\title{
Promoção da saúde do trabalhador: análise das ações propostas por concluintes de um curso de capacitação (2012)
}

\author{
Workplace health promotion: analysis of actions proposed by \\ graduates of a training course (2012)
}

\author{
Francielle Bertusso'; Manoela de Carvalho²; Marcelo de Souza Furtado ${ }^{3}$; \\ Neide Tiemi Murofuse,
}

\begin{abstract}
Resumo
A incorporação do conhecimento do trabalhador nas ações de promoção e prevenção da saúde pode potencializar a luta por melhores condições de trabalho e a defesa da saúde do trabalhador. Este estudo bibliográfico teve como objetivo analisar, a partir do referencial teórico da saúde do trabalhador, as ações propostas pelos concluintes de um curso de capacitação para a promoção da saúde do trabalhador da saúde, em 2012. Do total de 221 projetos analisados, os trabalhadores de saúde constituíram o públicoalvo prioritário (84\%), além dos gestores $(12 \%)$, oriundos de várias regiões do país com predomínio das propostas das regiões sudeste (107 projetos) e nordeste (55 projetos), e com atuação em diversos locais de trabalho. As propostas de intervenção intentaram resolver ou minimizar três conjuntos de problemas: a) relacionados ao ambiente de trabalho, b) a gestão do trabalho e c) a saúde do trabalhador. A análise das propostas apontou para uma realidade de trabalho permeada por ocorrência de acidentes de trabalho por exposição a material biológico, perfurocortante, radiação ionizante e de agravos relacionados a sobrecarga osteomuscular, relacionados a saúde mental como o transtorno mental e comportamental, depressão, alcoolismo além de estresse, burnout e assédio moral. Entre as ações de enfrentamento constatou-se a presença significativa de propostas conformadas nos moldes das abordagens tradicionais da saúde e segurança no trabalho, junto com outras sustentadas nos pressupostos do campo da Saúde do Trabalhador. Considerando o referencial do campo da saúde do trabalhador, conclui-se que permanece o desafio pela superação da abordagem das relações trabalho e saúde-doença fundamentado na ideia cartesiana do corpo como máquina, vinculado à medicina do trabalho e saúde ocupacional, cujas propostas sugerem a supressão de agentes/fatores de risco ambiental como meio de promover a saúde no trabalho.
\end{abstract}

Palavras-chave: Saúde do trabalhador. Pessoal de saúde. Promoção da saúde. Capacitação profissional.

\begin{abstract}
The incorporation of the workers knowledge in the actions of health promotion and prevention can strengthen the search for better working conditions and the protection of workers' health. This bibliographic study aimed to analyze, from the theoretical framework of occupational health, the actions proposed by graduates of a training course for health promotion for healthcare workers in 2012. Of the 221 projects analyzed, health workers were the priority target audience $(84 \%)$, as well as managers $(12 \%)$, from various regions of the country with a predominance of proposals from the southeast region (107 projects) and northeast region (55 projects), and in several workplace activities. Program proposals attempted to solve or minimize three sets of

\footnotetext{
${ }^{1}$ Enfermeira, aluna especial do Programa de Mestrado em Biociências e Saúde da Universidade Estadual do Oeste do Paraná, campus de Cascavel. E-mail: franbertusso@hotmail.com

${ }^{2}$ Doutora em Saúde Coletiva, Professora adjunta do Curso de Enfermagem do Centro de Ciências Biológicas e da Saúde da Universidade Estadual do Oeste do Paraná, campus de Cascavel, Pr.

${ }^{3}$ Enfermeiro da Secretaria de Estado da Saúde do Paraná - SESA. Aluno especial do Programa de Mestrado em Biociências e Saúde da Universidade Estadual do Oeste do Paraná, Campus de Cascavel, Pr.

${ }^{4}$ Doutora em Enfermargem pela Universidade de São Paulo. Professora do Programa de Mestrado em Biociências e Saúde do Centro de Ciências Biológicas e da Saúde da Universidade Estadual do Oeste do Paraná, campus de Cascavel
} 
problems: a) related to the work environment, b) labor management and c) the workers' health. Examination of the proposals pointed to a working reality permeated by incidents of accidents by exposure to biological material, ionizing radiation, needlestick and sharps injuries, and injuries related to musculoskeletal overload, mental health and mental and behavioral disorder such as depression, alcoholism, stress, burnout and moral harassment. Amongst the actions of confrontation, the significant presence of shaped proposals along the lines of traditional approaches to health and safety at work was observed, along with other sustained in the field assumptions of workers' health. Considering the framework of occupational health field, it is concluded that the challenge remains for overcoming the approach of working relations and health and disease based in the cartesian idea of the body as machine, linked to occupational medicine and occupational health, whose proposals suggest the suppression of agents / environmental risk factors as a means of promoting health.

Key words: Occupational health. Health personnel. Health promotion. Professional training.

\section{Introdução}

A crise atual do capital nada mais é do que o modo natural de existência do capital, buscando impor um novo padrão de acumulação capitalista em escala planetária. Para reconstituir sua base de produção, exploração da força de trabalho e acumulação de valor, o capital promove alterações na organização e gestão do trabalho para romper com a barreira que dificultava a exploração da força de trabalho e facilitar a precarização do trabalho vivo produzindo o que foi denominado por Alves (2011) como o sociometabolismo da barbárie, um novo metabolismo social resultado das novas condições históricas de reprodução expandida do capital, como um dos elementos da gênese da debilitação do movimento social do trabalho e da crise do sindicalismo nos principais países capitalistas.

A acumulação flexível caracterizada como um confronto com a rigidez do fordismo surge como estratégia corporativa para enfrentar as condições crítica da etapa do capitalismo marcado pela mundialização financeira e crise de sobreacumulação, por meio da flexibilização das condições de produção, principalmente da força de trabalho (ALVES, 2011). Poupado quantitativamente e explorado qualitativamente de forma mais intensa, observa-se a proliferação do subemprego, do trabalho precário e informal. A denúncia sobre o crescente adoecimento físico e mental dos trabalhadores tem sido constante, especialmente, nas últimas décadas, sendo estes agravos relacionados com a reestruturação produtiva do trabalho e a precarização das condições de trabalho as quais tem o aumento da intensidade e das exigências no trabalho como marcas fundamentais (MINAYO; MACHADO; PENA, 2011).

As mudanças tecnológicas e organizacionais ocorreram no mundo do trabalho em geral, incluído o setor saúde, que tem como uma de suas características, a presença intensiva e uma forte dependência do trabalho humano; com 3.078.518 trabalhadores da saúde, correspondendo a 4,3\% da população ocupada no Brasil (ASSUNÇÃO, 2012) apresentando uma dinâmica que o distingue dos demais setores econômicos, especialmente pela centralidade do trabalho vivo (DEDECCA; TROVÃO, 2013).

Dedecca e Trovão (2013) indicam que o complexo da saúde vem expandindo em termos ocupacionais, independente da conjuntura econômica, motivado pela ampliação do atendimento público à saúde, pela diversificação da cesta de serviços, a expansão da cobertura, reforçado pelo envelhecimento da população, marcado pela recorrente restrição dos recursos financeiros que tem resultado na deterioração das condições e relações de trabalho. Acrescentam, ainda, este complexo tem um incremento lento da produtividade, ao contrário do observado em outros setores da atividade econômica, num mercado de trabalho no sistema (público e privado) com tendência a continuidade do assalariamento e da precarização das relações de trabalho, fruto da desregulamentação do mercado implementado pelas políticas neoliberais, subtraindo direitos trabalhistas, 
gerando insegurança e insatisfação entre profissionais e trabalhadores desta área.

As questões referentes ao mundo do trabalho e suas repercussões na saúde dos trabalhadores, de modo particular aqueles relacionados a saúde, tem despertado o interesse de estudiosos de várias áreas, em âmbito nacional e internacional, contribuindo para o entendimento de diversos aspectos, entre eles: dos acidentes e doenças do trabalho (BARBOSA; ASSUNÇÃO; ARAÚJO, 2012; MARTINS; SILVA; CORREIA, 2012; OLIVEIRA; PAIVA, 2013), da saúde mental (DILÉLIO et al., 2012; RESENDE et al., 2011), das condições de trabalho (ISOSAKI et al,. 2011; SHIMIZU; CARVALHO JUNIOR, 2012), qualidade de vida (ANDRADE; ALBUQUERQUE; ANDRADE, 2011; TSA et al., 2012), do presenteísmo, fenômeno estudado mais recentemente, presente nas organizações de trabalho mas ainda pouco visível e que interfere negativamente no desempenho do trabalhador que comparece ao trabalho mesmo doente ou com problema físico ou psicológico (UMANN; GUIDO; GRAZZIANO, 2012) e das relações laborais no setor público de saúde sob o foco do direito no trabalho e ao trabalho (MARTINS; MOLINARO, 2013).

A nova conformação do mundo do trabalho e a fragilidade dos sindicatos de trabalhadores dificultam e impedem, em grande medida, o desenvolvimento da capacidade protetora de agravos à saúde e acidentes e doenças dos trabalhadores, como preconiza a abordagem em Saúde do Trabalhador, já que eles não conseguem intervir e transformar a realidade de trabalho, por meio da participação e controle das nocividades, do planejamento e decisão consensual de prioridades de intervenção e das estratégias transformadoras (COSTA et al., 2013).

Neste sentido, a Saúde do Trabalhador (ST), campo de práticas e conhecimentos que emerge da Saúde Coletiva, sofre um retrocesso e possibilita o fortalecimento das abordagens da relação trabalho e saúde da Saúde Ocupacional (SO) e da Medicina do Trabalho (MT), às quais pretendem se contrapor e superar. Enquanto a ST busca abordar a relação trabalho-saúde a partir da interdisciplinaridade fundamentada em uma compreensão da determinação social do processo saúde-doença da classe trabalhadora, a Saúde Ocupacional:

[...] incorpora práticas e conhecimentos da clínica, medicina preventiva e epidemiologia clássica, mediante a história natural da doença para a análise das doenças e acidentes do trabalho mediante a tríade 'agente-hospedeiro-ambiente', conforme proposto em 1950 pelo Comitê Misto de Peritos da Organização Internacional do Trabalho (OIT)/ Organização Mundial da Saúde (LACAZ, 2007, p. 758).

A abordagem da Medicina do Trabalho nasce na Inglaterra no século XIX, a partir da implantação dos serviços médicos dentro das fábricas, tendo o médico como responsável pela prevenção de acidentes e doenças dos empregados, com base na teoria unicausal do adoecimento. A preocupação principal era a produtividade e não a promoção da saúde do trabalhador, sendo este tratado como mero objeto de trabalho (SELIGMANN-SILVA et al., 2010). O incremento da Medicina do Trabalho decorreu da necessidade surgida, com a implementação da produção em série e aumento da produtividade, de operário sadio, com baixo índice de absenteísmo e alta produção. E ao médico cabia selecionar os mais aptos o trabalho e o atendimento do trabalhador, nas dependências da própria empresa, para que o mesmo pudesse retornar, sem demora, à linha de montagem. Muito embora a Saúde Ocupacional tenha representado um avanço em relação ao período da Medicina do Trabalho, o enfoque multiprofissional não foi capaz de diminuir os altos índices de acidentes do trabalho, principalmente nos países em desenvolvimento (OLIVEIRA, 2010).

Nesta perspectiva, a busca de medidas protetivas e preventivas capazes de melhorar as condições de trabalho e da saúde implica enfrentar os conflitos e contradições claras de interesses, considerando que as relações de trabalho-saúde 
situam-se na luta política de interesses antagônicos e irreconciliáveis entre o capital e o trabalho em sociedades concretas. As exigências em defesa da saúde do trabalhador devem ser compreendidas como principal agenda das lutas dos trabalhadores, dos profissionais e dos gestores das políticas sociais, para que, se tenha saúde para o trabalho e para a vida. No Brasil, algumas ações sociais estão integradas na luta da promoção e preservação da saúde dos trabalhadores, a partir de iniciativas de movimentos sociais e algumas instituições, cada uma contando com instrumentos e competências específicas, produzindo experiências importantes na Saúde do Trabalhador(LIMA, 2009). Entretanto, existe uma lacuna importante de estudos acerca das concepções e do posicionamento político dos profissionais e trabalhadores de saúde, em relação à sua prática e em relação ao sistema e à política de saúde, em particular, da área da ST.

Combasenesses pressupostos eno entendimento de que a incorporação do conhecimento dos trabalhadores pode potencializar as lutas pela melhoria das condições de trabalho e da defesa pela vida, o presente estudo teve como objetivo analisar as ações propostas pelos concluintes de um curso de capacitação com vistas a promoção da saúde do trabalhador da saúde, buscando identificar a filiação das mesmas a uma das três abordagens da relação trabalho-saúde: a Medicina do Trabalho, a Saúde Ocupacional e a Saúde do Trabalhador.

\section{Metodologia}

Foi desenvolvido uma pesquisa bibliográfica com uma abordagem qualitativa dos dados. Por meio da consulta online ao banco de dados da biblioteca virtual de uma universidade pública brasileira foi localizado um total de 233 projetos. Após a exclusão de 12 propostas devido a repetição ou a impossibilidade de acesso por falha no endereçamento eletrônico foram coletadas informações referentes a 221 projetos apresentados como trabalhos de conclusão (TCC) no ano de 2012, de um curso de atualização semipresencial que teve como tema as condições de trabalho e saúde do trabalhador da saúde. O curso de atualização analisado foi ofertado em parceria estabelecida entre uma universidade pública, o Ministério da Saúde, representada pelo Departamento de Gestão e Regulação do Trabalho em Saúde (DGERT) e a Organização Pan-Americana de Saúde (OPAS) e teve como objetivo qualificar os profissionais vinculados à gestão do Sistema Único de Saúde (SUS) para a formulação e execução de políticas de proteção ao trabalho, bem como os trabalhadores do setor saúde e propôs como TCC, a elaboração de projetos de intervenção nos locais de trabalho dos participantes do curso.

Após a identificação do material bibliográfico para o registro dos dados coletados utilizouse de um formulário elaborado pelos autores contendo: a) os problemas-alvo e a motivação dos participantes do curso, b) os objetivos propostos, c) a população alvo, d) tipo de instituição e e) região geográfica das propostas apresentadas nos projetos de TCC. A coleta foi realizada durante o período compreendido entre os meses de maio a julho de 2013.

Este estudo utilizou como fonte de dados informações disponibilizadas na biblioteca virtual disponível no site da universidade pública, com acesso público e sem restrição. Assim, este artigo apresenta análises a partir de conteúdo de domínio público, que não exige da tramitação em Comitês de Ética em Pesquisas com seres humanos, conforme legislação. No entanto, buscou-se cumprir os preceitos éticos do anonimato, o sigilo e a não maleficência, sendo ocultadas informações que pudessem identificar a origem da pesquisa e dos pesquisados, resultantes da coleta.

Para facilitar a análise os dados coletados foram digitados num banco de dados construído segundo 
codificação previamente definida, utilizando-se do programa Microsoft Excel. Depois de constituído o banco de dados, os conteúdos extraídos dos projetos foram submetidos a leitura e releituras do material acompanhado da anotação das primeiras impressões no sentido de captar as ideias centrais. $\mathrm{Na}$ sequência para agrupar os conteúdos similares houve um processo classificatório em que as unidades de sentido, os temas, foram colocadas juntas. E, na terceira e última etapa, foi efetuado o refinamento do movimento classificatório (MINAYO, 2008) do qual se obteve dois conjuntos, como pode ser visualizado por meio do quadro 1 apresentado a seguir:

Quadro 1 - Agrupamento dos temas e sub-temas extraídos dos projetos analisados, 2012

\begin{tabular}{|l|ll|}
\hline TEMA & \multicolumn{2}{|c|}{ SUB TEMA } \\
\hline $\begin{array}{l}\text { 1) problemas enfrentados } \\
\text { pelos concluintes }\end{array}$ & $1.1)$ & relacionados ao ambiente de trabalho \\
& 1.2) & relacionados a gestão do trabalho \\
\hline $\begin{array}{l}\text { objetivos das ações } \\
\text { propostas }\end{array}$ & 2.1) gestão do trabalho \\
& 2.2) saúde do trabalhador \\
\hline
\end{tabular}

Fonte: Dos autores.

Os conteúdos das temáticas foram analisados e interpretados tendo como base a produção científica específica da área, os princípios, diretrizes, conceitos e a legislação que respaldam e norteiam a prática na área da Saúde do Trabalhador, da Saúde Ocupacional e da Medicina do Trabalho.

\section{Resultados e Discussão}

Os trabalhadores de saúde constituíram o público-alvo prioritário dos projetos ( $84 \%)$, além dos gestores (12\%), oriundos de várias regiões do país com predomínio das propostas das regiões sudeste (107 projetos) e nordeste (55 projetos), e com atuação em diversos locais de trabalho como: instituições assistenciais (ambulatórios, unidades básicas de saúde, laboratório municipal, posto de assistência médica, unidade de emergência, unidades de pronto atendimento, hemocentros, hospitais - geral, universitário, emergência e urgência, regional e municipal - institutos de cardiologia, centros de atenção psicossocial, centros de referencia em saúde do trabalhador e clínica infantil), instituições de ensino e pesquisa (escola de saúde pública, instituição pública de ensino e pesquisa) e administrativas (distrito sanitário, secretarias municipal e estadual de saúde).

De acordo com os projetos analisados os problemas vivenciados nos locais de trabalho dos concluintes do curso estariam circunscritos a três conjuntos de aspectos, apresentados no quadro 2 , a seguir.

Quadro 2 - Propostas de intervenção apresentados pelos concluintes do curso de atualização, 2012

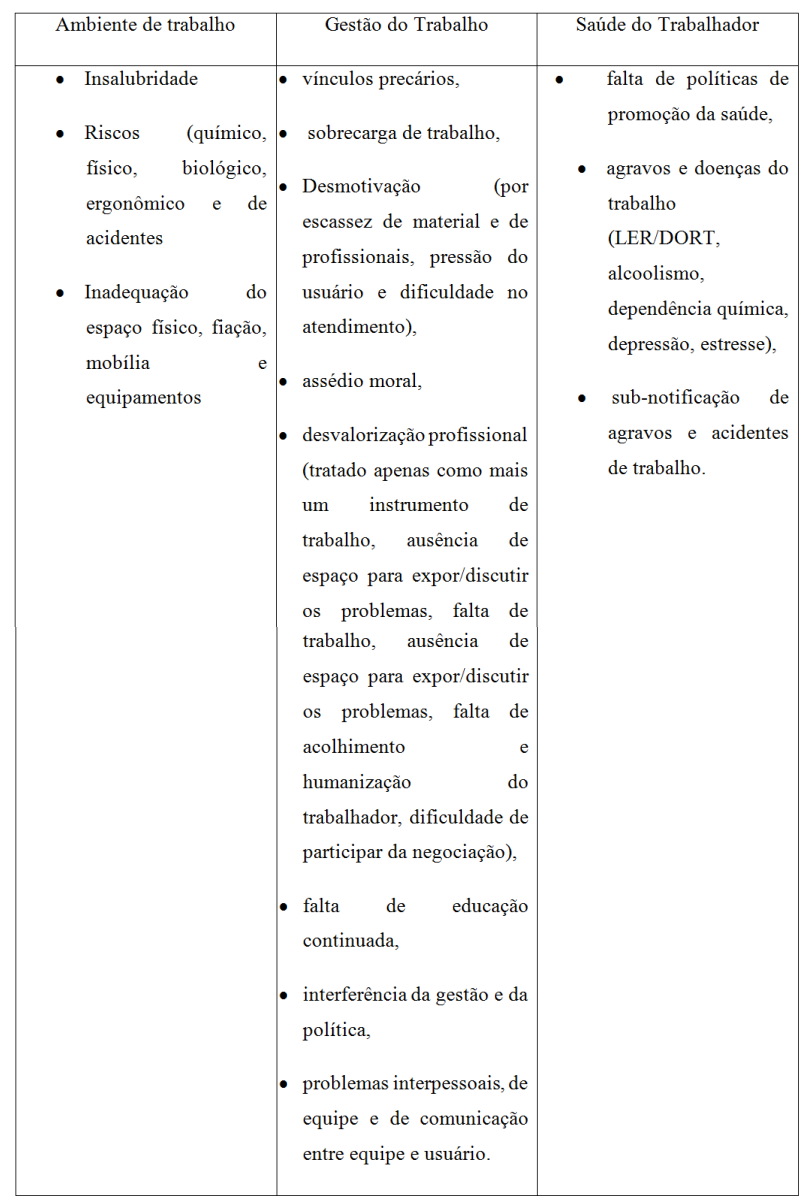

Fonte: Dos autores. 
O conjunto de problemas elencados no Quadro 2 é a expressão de uma realidade permeada por antigos e novos problemas que podem consumir a força de trabalho em saúde, resultando em problemas de saúde que afetam corpos e mentes, denunciando tanto a violência explícita (acidentes e doenças do trabalho) quanto a violência oculta no trabalho manifestadas pelas chamadas doenças da modernidade como as LER/DORT, depressão, angústia, estresse, alcoolismo, dentre outras (HANDAR, 2012). Além das consequências para a saúde do trabalhador, este tipo de ambiente de trabalho é responsável por ausências no trabalho, como bem ilustra os resultados do estudo sobre absenteísmo realizado por Fernandes et al. (2011), além das repercussões que afetam usuários, familiares e a instituição de saúde.

Como já assinalado anteriormente por Dedecca e Trovão (2013) o trabalho em saúde tem como característica a centralidade do trabalho vivo distinguindo assim dos demais setores econômicos, vem sofrendo com a recorrente restrição dos recursos financeiros que tem resultado na deterioração das condições de trabalho e da precarização das relações de trabalho, fruto das políticas neoliberais que vem desregulamentado o mercado. O neoliberalismo entendido como um paradigma econômico e político também é causa de impactos na área da saúde, especialmente com a reuniversalização da saúde através de uma vertente predominantemente economicista que tem resultado em desregulamentação, privatizações e aberturas de mercado tornando assim, a saúde, um bem comum, apenas um privilegio daqueles que podem “consumir" a mercadoria saúde.

Para o enfrentamento dos problemas foi proposto dois conjuntos de ações extraídos dos objetivos dos projetos e apresentados no Quadro 3, a seguir. As propostas referentes aos aspectos da Saúde do Trabalhador e da Gestão do Trabalho, separadas apenas para facilitar a visualização, constituem aspectos de uma mesma realidade da prática profissional em saúde estando intimamente interconectados e interdependentes.
Quadro 3 - Objetivos das propostas de intervenção apresentados pelos concluintes do curso de atualização, 2012

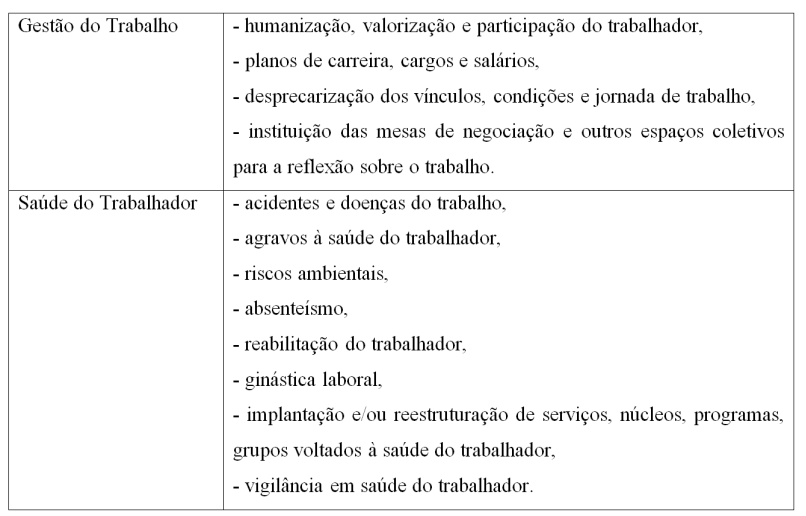

Fonte: Dos autores.

O trabalho em saúde atualmente tem sido foco de estudo e atenção, especialmente, pela crescente precarização das condições de trabalho e suas repercussões na qualidade de vida e saúde dos trabalhadores. E, esta situação ficou evidenciada também com a análise dos objetivos das propostas de intervenção apresentadas pelos alunos concluintes do curso estudado (Quadro 3), visando enfrentar problemas na dimensão micro do processo de trabalho, que afetam o cotidiano das equipes de saúde. Atualmente, os novos modelos de atenção e gestão tem proposto (e "exigido") que os trabalhadores da saúde sejam mais participativos, porém, na prática, tem sido inviabilizado ou limitado o efetivo exercício dessa condição de sujeito do processo de trabalho, como, aponta o conjunto dos objetivos relativo à gestão do trabalho.

No que se refere ao conjunto dos objetivos das propostas de intervenção analisados referente ao grupo denominado "saúde do trabalhador" (Quadro3) constata-se o que foi pontuado por Sato (2012) como sendo negociações para operar micromudanças no cotidiano de trabalho na tentativa de amenizar o sofrimento, o incômodo e os esforços sentido pelos trabalhadores no seu dia-a-dia, as quais apresentam limites postos pelo próprio contexto no qual se dão. Entre as ações de enfrentamento constatou-se a presença significativa de propostas 
conformadas nos moldes das abordagens tradicionais da saúde e segurança no trabalho, junto com outras sustentadas nos pressupostos do campo da Saúde do Trabalhador. Ao analisar os propósitos das propostas apresentadas pelos concluintes do curso constatou-se que houve um descompasso em relação à indicação dada pela organização do curso para que as ações propostas para o enfrentamento dos problemas oriundos da prática fossem elaboradas em conformidade com as Diretrizes da Política Nacional de Promoção da Saúde do Trabalhador do SUS (ASSUNÇÃO, 2012). Observa-se que foram mais significativas as propostas conformadas nos moldes do que foi denominado no documento do Ministério da Saúde (BRASIL, 2011) como abordagens tradicionais da saúde e segurança no trabalho, consideradas ultrapassadas quando se intenta a promoção da saúde na perspectiva das Diretrizes. As propostas para "implantação de mapa de riscos ambientais e sensibilização das equipes", "conscientização do trabalhador para o uso de luvas e a lavagem correta das mãos", "conscientizar sobre a importância da realização dos exames periódicos", "melhorar a adesão dos profissionais às medidas de biossegurança", "implantação de um serviço de Saúde Ocupacional", "reestruturação do Serviço Especializado em Segurança e Medicina do Trabalho (SESMT)", "fortalecimento e instituição da Comissão Interna de Prevenção de Acidentes (CIPA) em serviço público", "formação de equipe multiprofissional de saúde do trabalhador", são exemplos de formulações condizentes com a abordagem da medicina preventiva, de onde emerge a abordagem da Saúde Ocupacional.

No Brasil, a adoção e o desenvolvimento da Saúde Ocupacional se deu em diversos âmbitos, incluindo a legislação, expresso nas Normas Regulamentadoras (NRs), no capítulo $\mathrm{V}$ da Consolidação das Leis do Trabalho (CLT) como, por exemplo, a obrigatoriedade de equipes técnicas multidisciplinares nos locais de trabalho, na avaliação quantitativa de riscos ambientais e adoção de limites de tolerância. A criação dos SESMT, decorrente da determinação de uma política adotada pelo regime militar que institucionalizou nacionalmente, nos anos 1970, as teorias científicas hegemônicas e repassou às empresas a tutela da saúde do trabalhador com vistas ao aumento da produtividade (OLIVEIRA, 2010).

O trabalho insalubre é caracterizado pela norma jurídica brasileira (art. 189, da CLT) quando ocorre a conjugação da exposição do trabalhador a agentes nocivos à saúde e a violação dos limites de tolerância, entendida como a concentração ou intensidade máxima ou mínima, relacionada com a natureza e o tempo de exposição ao agente, que não causará dano à saúde do trabalhador, durante a sua vida (NR-15, tópico 15.1) (BRASIL, 1978). Oliveira (2010) compara diversos ordenamentos jurídicos do mundo e indica três estratégias possíveis diante dos agentes agressivos: a) aumentar a remuneração para compensar o maior desgaste do trabalhador (monetização do risco); b) proibir o trabalho; c) reduzir a duração da jornada. A legislação brasileira opta pela monetização do risco e cria o pagamento de adicional em percentual conforme a classificação do grau de exposição ao agente para compensar o maior desgaste e lesão à saúde do trabalhador. Aprofunda, ainda mais, as raízes da monetização do risco, ao instituir a aposentadoria especial aos trabalhadores em serviços penosos, insalubres ou perigosos, incentivando o desempenho de atividades de risco com o aumento da remuneração (adicionais) e a concessão precoce de aposentadoria.

O fenômeno da mundialização do capital também repercute no plano jurídico-trabalhista, especialmente, pela implicação dos custos da mão de obra, direitos e contribuições sociais na concorrência intercapitalistas. Oliveira (2010) aponta como tendências atuais: a) o propósito de eliminar os riscos para a saúde do trabalho na sua origem, ao invés de tentar neutralizá-los com o uso de EPIs ou diminuir a jornada de trabalho com o intuito de conceder ao trabalhador maior período de descanso ou recuperação, b) banir "a solução retrógrada de compensar a agressão por adicionais 
(monetização do risco)" e aumentar a dignificação no trabalho, rechaçando a política de remunerar as agressões à saúde, que acelera o desgaste do trabalhador e apressa a sua morte.

A fixação dos adicionais de risco tinha como finalidade aumentar a remuneração do trabalhador e, desta forma, forçar o empregador a eliminar os elementos nocivos à saúde do trabalhador. $\mathrm{Na}$ prática, porém, observa-se a eternização do pagamento do adicional e os efeitos prejudiciais da monetização das atividades de risco afetando a segurança e saúde do trabalhador individual, coletiva e socialmente como expressam as enfermidades (físicas, psíquicas), mutilações ou mortes dos trabalhadores. Portanto, o desempenho de atividades de risco viola a dignidade humana, do direito à vida, o direito à saúde e o direito ao ambiente de trabalho saudável bem como resulta no aumento do custo com as aposentadorias precoces, a maior ocupação das redes de serviços de saúde (públicas e privadas) e aumenta o ônus de toda a sociedade com a saúde e a seguridade social.

As propostas apresentadas pelos concluintes do curso sobre a necessidade de mapear os riscos ambientais bem como a constituição das CIPAs estão respaldadas na legislação brasileira, especialmente nas Normas Regulamentadoras - NRs, dirigidas aos trabalhadores com vínculo de trabalho regido pela CLT. Uma das críticas sobre a legislação trabalhista enfatiza que ao atribuir a causa dos acidentes de trabalho aos riscos ambientais, está se contribuindo para a "naturalização dos riscos" bem como aceita como sendo inevitável a mudança no modo como está determinada a organização do trabalho (OLIVEIRA, 2007). O setor saúde também está contemplado nas NRs com uma legislação federal específica a NR-32, aprovada em 16 de novembro de 2005 (BRASIL, 2005). Tal Norma em suas diretrizes básicas de segurança e saúde no trabalho indicam formas de proteger a saúde do trabalhador agrupadas em três eixos: (a) a capacitação contínua dos trabalhadores seguida pela (b) definição e desenvolvimento de programas que tratam dos riscos e por último, (c) utilização de medidas técnicas de proteção contra os riscos. Para a prevenção de acidentes e doenças do trabalho estabelece dois tipos de medidas técnicas para o controle dos riscos ambientais: o primeiro por meio do uso de equipamento de proteção individual (EPI), para evitar ou diminuir a lesão e o segundo por meio dos equipamentos de proteção coletiva (EPC) com a finalidade de sinalizar, neutralizar ou eliminar o risco com o foco no ambiente.

Na prática cotidiana da saúde, os acidentes de trabalho com material biológico e perfurocortantes ainda são frequentes; o que também foi confirmado pelos concluintes do curso analisado ao ser considerado como um problema a ser enfrentado nos seus locais de trabalho, por meio das várias ações propostas: melhorar a adesão dos profissionais às medidas de biossegurança, capacitação das equipes, sensibilização dos profissionais, trabalhadores e gestores quanto a importância da prevenção e da notificação dos acidentes, educação permanente para a prevenção, proposta de cooperação da CIPA e do Serviço Especializado de Segurança e Medicina do Trabalho (SESMT) na prevenção de acidentes, prevenção da hepatite B por meio da imunização.

Atribuir ao conhecimento deficiente como sendo a causa da falta de adesão dos trabalhadores da saúde às medidas de biossegurança, deixa de levar em consideração outros possíveis motivos envolvidos tais como: desconforto e a inadequação do tipo de equipamento disponibilizado, a intensidade e o volume das atividades, a carga de trabalho e o reduzido quantitativo de pessoal, a jornada extensa de trabalho, entre outros. Em relação à proposta de uma prática preventiva baseada na busca da "conscientização" do trabalhador sobre a importância da adesão/adoção de algum equipamento/comportamento cabe ressaltar a parcialidade da medida que pouco contribui para a efetiva prevenção, pois se aproxima daquela explicação de acidente baseado na dicotomia entre atos e condições inseguras; causada por uma cadeia linear de fatores culminando em lesão. Além disso, responsabiliza o trabalhador pelo comportamento 
que se julga inadequado, impondo o fenômeno da culpabilização ao próprio trabalhador pelo acidente do qual, na realidade é vítima.

A atuação com enfoque sobre os indivíduos, no sentido de educar o trabalhador para que assimilem a "cultura" prevencionista, aliada ao paradigma da causalidade dos agravos à saúde por meio da identificação dos riscos, influi na compreensão do nexo entre trabalho e processo saúde-doença das abordagens utilizadas pela Medicina do Trabalho e pela Saúde Ocupacional (LACAZ, 2007). Diferentemente, a Saúde do Trabalhador compreende não existir riscos 'naturais' e busca seus determinantes no processo e organização do trabalho. Visa, principalmente, a modificação dos ambientes de trabalho e da organização do trabalho por meio de ação conjunta com os trabalhadores, sujeitos do processo, e não apenas objeto da atenção de técnicos como concebido pelas outras abordagens. Na questão da prevenção/promoção, enfatiza as ações coletivas com o protagonismo dos trabalhadores, ao invés de atuar sobre indivíduos privilegiando apenas os problemas de natureza orgânica/biológica.

Neste sentido, na busca de medidas de prevenção mais efetivas e a promoção da saúde do trabalhador, ao incorporar conhecimentos dos trabalhadores, a Saúde do Trabalhador torna mais consistente a luta por melhorias das condições de trabalho e defesa da vida. Visa compreender o processo saúdedoença dos grupos humanos em sua relação com o trabalho, levando em conta a atual complexidade que envolve a organização do trabalho e sua relação com a subjetividade de coletivos de trabalhadores, contribuindo desta maneira para a compreensão da gênese das doenças do trabalho, especialmente as emocionais e mentais, características do adoecimento do trabalho de hoje.

Dentre as ações propostas nos projetos analisados, constataram-se aspectos que se aproximam dos pressupostos que sustentam o campo da Saúde do Trabalhador como a da participação dos trabalhadores, controle e avaliação, acesso às informações, vigilância nos locais de trabalho geradores de danos. Houve propostas de ações no sentido de atender ao trabalhador acidentado ou doente como: a elaboração de fluxos tanto para a notificação quanto para o atendimento dos acidentes, a análise epidemiológica dos agravos à saúde, análise das notificações no SINAN-NET dos acidentes com material biológico, elaboração de protocolo e assistência integral ao trabalhador acidentado, análise e desenvolvimento de sistemas de registro do absenteísmo segundo o motivo, a implantação de programa de reabilitação profissional, readaptação funcional e de proteção à saúde do trabalhador deficiente. Houve ainda a proposição de ações alinhadas às legislações em ST tais como: 'implantação do Centro de Referencia em Saúde do Trabalhador (CEREST)', 'implantação de programas de vigilância à saúde do trabalhador', 'reestruturação da rede sentinela em saúde do trabalhador', entre outros.

As novas formas de adoecimento decorrentes das condições de trabalho, também motivaram propostas de intervenção dos projetos analisados, tais como: 'elaboração de diagnóstico, de forma coletiva, das causas dos transtornos mentais e depressão', 'rodas de conversa sobre a organização do trabalho e psicoestressores', 'promoção da saúde mental dos servidores públicos', 'assistência ao trabalhador com estresse ocupacional', entre outros. Neste sentido, conhecer os perfis de acidentes e doenças do trabalho dos trabalhadores da saúde pode ser um indicador para instrumentalizar o controle social e possibilitar o questionamento da responsabilidade das organizações e instituições com a saúde dos seus trabalhadores.

\section{Conclusões}

O estudo realizado possibilitou evidenciar um conjunto de problemas com consequências para a saúde tanto no aspecto físico quanto emocional dos trabalhadores de diferentes instituições 
de saúde assistenciais, de ensino e pesquisa e administrativas, de 27 estados das cinco regiões do Brasil. Além da exposição a acidentes envolvendo material biológico, sobrecarga osteomuscular, depressão, alcoolismo, burnout e assédio moral houve propostas com a intenção de resolução de problemas relacionados à gestão do trabalho tais como a humanização, valorização e participação do trabalhador, a implantação do plano de carreira, cargos e salários, a desprecarização dos vínculos, condições e jornada de trabalho, a instituição das mesas de negociação e outros espaços coletivos para a reflexão sobre o trabalho. Portanto, indica uma realidade de trabalho em saúde permeado por velhos e novos problemas além da exigência de uma atuação mais participativa dos trabalhadores num contexto em que restringe e limita a efetiva atuação dos mesmos.

Constatou-se a presença entre as propostas de enfrentamento dos problemas de concepções que responsabilizam os próprios trabalhadores pelos acidentes e doenças pelos quais na realidade são vítimas e não culpados. Considerando o referencial do campo da saúde do trabalhador, concluise que permanece o desafio pela superação da abordagem das relações trabalho e saúde-doença, fundamentado na ideia cartesiana do corpo como máquina, vinculado à medicina do trabalho e saúde ocupacional, cujas propostas sugerem a supressão de agentes/fatores de risco ambiental como meio de promover a saúde no trabalho. Portanto, ainda se mantém o desafio de superar as abordagens tradicionais da saúde e segurança do trabalho pela efetiva implementação de ações fundamentadas nas concepções da Saúde do Trabalhador.

\section{Referências}

ALVES, G. Trabalho e subjetividade: o espírito do toyotismo na era do capitalismo manipulatório. São Paulo: Boitempo, 2011.
ANDRADE, A. N. M.; ALBUQUERQUE, M. A. C.; ANDRADE, A. N. M. Avaliação do nível de estresse do anestesiologista da Cooperativa de Anestesiologia de Sergipe. Revista Brasileira de Anestesiologia, Rio de Janeiro, v. 61, n. 4, p. 48694, 2011.

ASSUNÇÃO, A. Á. Gestão das condições de trabalho e saúde dos trabalhadores da saúde: caderno de estudos. Belo Horizonte: Nescon/ UFMG, 2012.

BARBOSA, R. E. C.; ASSUNÇÃO, A. Á.; ARAÚJO, T. M. Distúrbios musculoesqueléticos em trabalhadores do setor saúde de Belo Horizonte, Minas Gerais, Brasil. Cadernos de Saúde Pública, Rio de Janeiro, v. 28, n. 8, p. 1569-1580, 2012.

BRASIL. Ministério do Trabalho. Portaria 3214 de 08 de junho de 1978- NR 15. Atividades e operações insalubres. Diário Oficial da União, Brasília, 6 jul. 1978. p. 33.

Portaria 485 de 11 de novembro de 2005. Aprova a Norma Regulamentadora $n^{\circ} 32$ (Segurança e Saúde no Trabalho em Serviços de Saúde). Diário Oficial da União, Brasília, 16 nov. 2005.

Ministério da Saúde. Secretaria de Gestão do Trabalho e da Educação na Saúde. Secretaria de Vigilância em Saúde. Mesa Nacional de Negociação Permanente do SUS. Protocolo $N^{o} 008 / 2011$ da Mesa Nacional de Negociação Permanente do Sistema Único de Saúde. Brasília: Ministério da Saúde, 2011.

COSTA, D.; LACAZ, F. A. C.; JACKSON FILHO, J. M.; VILELA, R. A. G. Saúde do trabalhador no SUS: desafios para uma política pública. Revista Brasileira de Saúde Ocupacional, Rio de Janeiro, v. 38, n. 127, p. 11-30, 2013.

DEDECCA, C. S.; TROVÃO, C. J. B. M. A força de trabalho no complexo da saúde: vantagens e desafios. Ciência e Saúde Coletiva, Rio de Janeiro, v. 18, n. 6, p. 1555-1567, 2013.

DILÉLIO, A. S.; FACHINI, L. A.; TOMASI, E.; SILVA, S. M.; THUMÉ, E.; PICCINI, R. X.; SILVEIRA, D. S.; MAIA, M. F. S.; OSÓRIO, A.; SIQUEIRA, F. V.; JARDIM, V. M. R.; LEMÕES, 
M. A. M.; BORGES, C. L. S. Prevalência de transtornos psiquiátricos menores em trabalhadores da atenção primária à saúde das regiões Sul e Nordeste do Brasil. Cadernos de Saúde Pública, Rio de Janeiro, v. 28, n. 3, p. 503-514, 2012.

FERNANDES, R. L.; HADDAD, M. C. L.; MORAIS, A. E. P.; TAKAHASHI, I. T. M. Absenteísmo em hospital filantrópico de médio porte. Semina: Ciências Biológicas e da Saúde, Londrina, v. 32, n. 1, p. 3-14, 2011.

HANDAR, Z. Agravos à saúde do trabalhador: processo saúde-doença-trabalho. In: CÊA, G. S. S; MUROFUSE, N. T.; DEITOS, R. A. Trabalho, educação e saúde: formação permanente de profissionais e usuários da saúde pública. Cascavel: Edunioeste, 2012.

ISOSAKI, M.; CARDOSO, E.; GLINA, D. M. R.; PUSTIGLIONE, M.; ROCHA, L. E. Intervenção nas situações de trabalho em um serviço de nutrição hospitalar e repercussões nos sintomas osteomusculares. Revista de Nutrição, Campinas, v. 24, n. 3, p. 449-462, 2011.

LACAZ, F. A. C. O campo Saúde do trabalhador: resgatando conhecimentos e práticas sobre as relações trabalho-saúde. Cadernos de Saúde Pública, Rio de Janeiro, v. 23, n. 4, p. 757-766, 2007.

LIMA, F. P. A. L. Ações coordenadas em saúde do trabalhador: uma proposta de atuação suprainstitucional. Revista Brasileira de Saúde Ocupacional, Rio de Janeiro, v. 34, n. 119, p. 67-78, 2009.

MARTINS, M. I. C.; MOLINARO, A. Reestruturação produtiva e seu impacto nas relações de trabalho nos serviços públicos de saúde no Brasil. Ciência Saúde Coletiva, Rio de Janeiro, v. 18, n. 6, p. 1667-1676, 2013.

MARTINS, M. D. S.; SILVA, N. A. P.; CORREIA, T. I. G. Acidentes de trabalho e suas repercussões num hospital ao Norte de Portugal. Revista LatinoAmericana de Enfermagem, Ribeirão Preto, v. 20, n. 2, p. 217-225, 2012.

MINAYO, M. C. S. O desafio do conhecimento: pesquisa qualitativa em saúde. 11. ed. São Paulo: Hucitec, 2008.

MINAYO, C. M.; MACHADO, J. M. H.; PENA, P. G. L. (Org.). Saúde do trabalhador na sociedade brasileira contemporânea. Rio de Janeiro: Fiocruz, 2011.

OLIVEIRA, A. C.; PAIVA, M. H. R. S. Análise dos acidentes ocupacionais com material biológico entre profissionais em serviços de atendimento pré-hospitalar. Revista Latino-Americana de Enfermagem, Ribeirão Preto, v. 21, n. 1, p. 309-315, 2013.

OLIVEIRA, F. A persistência da noção de ato inseguro e a construção da culpa: os discursos sobre os acidentes de trabalho em uma indústria metalúrgica. Revista Brasileira de Saúde Ocupacional, Rio de Janeiro, v. 32, n. 115, p. 19-27, 2007.

OLIVEIRA, S. G. Proteção jurídica à saúde do trabalhador. 5. ed. São Paulo: LTr, 2010.

RESENDE, M. C.; AZEVEDO, E. G. S.; LOURENÇO, L. R.; FARIA, L. S.; ALVES, N. F.; FARINA, N. P.; SILVA, N. C.; OLIVEIRA, S. L. Saúde mental e ansiedade em agentes comunitários que atuam em saúde da família em Uberlândia (MG, Brasil). Ciência e Saúde Coletiva, Rio de Janeiro, v. 16, n. 4, p. 2115-2122, 2011.

SATO, L. Saúde do trabalhador: objeto de negociação cotidiana. In: CÊA, G. S. S; MUROFUSE, N. T.; DEITOS, R. A. Trabalho, educação e saúde: formação permanente de profissionais e usuários da saúde pública. Cascavel, PR: Edunioeste, 2012. v. 2.

SELIGMANN-SILVA, E.; BERNARDO, M. H.; MAENO, M.; KATO, M. Saúde do trabalhador no início do século XXI. Revista Brasileira de Saúde Ocupacional, Rio de Janeiro, v. 35, n. 122, p. 1856, 2010.

SHIMIZU, H. E.; CARVALHO JUNIOR, D. A. O processo de trabalho na estratégia saúde da família e suas repercussões no processo saúde-doença. Ciência e Saúde Coletiva, Rio de Janeiro, v. 17, n. 9, p. 2397-2402, 2012. 
TSA, H. M. A.; OLIVERA, L. T.; BORTOLOZZO, C. R.; PETRY, S.; SCHUCH, T. F. Percepção da qualidade de vida entre médicos anestesiologistas e não anestesiologistas. Revista Brasileira Anestesiologia, Rio de Janeiro, v. 62, n. 1, p. 48-55, 2012.

UMANN, J.; GUIDO, L. A.; GRAZZIANO, E. $\mathrm{S}$. Presenteísmo entre enfermeiros hospitalares. Revista Latino-Americana de Enfermagem, Rio de Janeiro, v. 20, n. 1, p. 159-66, 2012. 